\title{
Measurement Adherence in the Blood Pressure Self-Measurement Room
}

Stefan Wagner, MS, ${ }^{1}$ Niels Henrik Buus, MD, PhD, DMSci, ${ }^{2}$

Bente Jespersen, MD, DMSci, ${ }^{2}$ Peter Ahrendt, $P h D,{ }^{1}$

Olav W. Bertelsen, $P h D_{1}^{3}$ and Thomas S. Toftegaard, $P h D^{1}$

Departments of ${ }^{1}$ Engineering and ${ }^{3}$ Computer Science,

Aarhus University, Aarhus, Denmark.

${ }^{2}$ Department of Renal Medicine, Aarhus University Hospital, Aarhus, Denmark.

\section{Abstract}

Background: Patients with hypertension or receiving blood pressure (BP)-lowering treatment are often required to self-measure their BP in a dedicated self-measurement room before consultation. Current praxis does not guarantee valid measurements, possibly leading to misdiagnoses or inappropriate antihypertensive medication. The aim of this study was to investigate patients' ability to correctly selfreport and follow recommendations. Patients and Methods: We used a context-aware system to gather information on BP measurements and relevant context parameters. Patients were not informed that the system automatically collected behavior data and were instructed to self-report their measurements on a paper sheet as usual. We then compared the automatically recorded data with the self-reported data in order to detect any nonadherent reporting behavior. Also, we investigated the patients' ability to adhere to the measurement recommendations. Results: We found that (1) a third of all 113 participating patients failed to self-report measured BP data correctly and (2) none of the 642 measurements obtained adhered fully to the recommendations. Conclusions: Results indicate that context-aware technology may be useful for accurately modeling aspects of nonadherent patient behavior. This may be used to inform staff of the validity of the measurement and pinpoint patients in need of additional training or to design better aids to assist the patients. The developed system is generally applicable to other self-measurement environments, including the home setting and remote outpatient clinics, as it is built using telemedicine technology and thus well suited for remote monitoring and diagnosis.

Key words: e-health, cardiology/cardiovascular disease, telemedicine, telehealth, home health monitoring

\section{Introduction}

$\mathrm{B}$ lood pressure (BP) measurements are important in the diagnosis and monitoring of patients suffering from hypertension or receiving BP-lowering medication. ${ }^{1-3} \mathrm{Hy}-$ pertension is estimated to be affecting a quarter of the world's adult population. ${ }^{4}$ Measurements can be performed at the clinic as an office BP measurement, as well as in the home. In the latter case, this may be done by using either ambulatory BP devices, which are typically worn by the user for a single 24-h diagnostic period, or automatic home BP devices for point measurements spanning several days. Both ambulatory and home devices have proven successful for obtaining valid measurements in clinical trials compared with office BP measurements, although home BP devices are both cheaper and easier to use than ambulatory BP devices. ${ }^{5,6} \mathrm{BP}$ self-measurement (BPSM) can also be performed by patients in outpatient clinics, as an alternative or supplement to home measurements, using the same BP device types and following the same techniques as in the home setting. ${ }^{4}$

Measurements at the clinic are believed to cause the "white coat effect," where patients are showing higher BP readings at the clinic than at home, possibly because of the anxiety some people experience during a visit to the clinic. This is estimated to affect as much as $20 \%$ of all patients. ${ }^{5,6}$

In the BPSM Room at the Department of Renal Medicine, Aarhus University Hospital, Aarhus, Denmark, patients are required to selfmeasure their BP before consultation. However, the BPSM process requires the patient to follow a range of recommendations to be valid, and even though careful instructions and training are provided, BPSM may still be associated with problems.

Current state-of-the-art BP devices used in the BPSM Room are not capable of sensing incorrect usage. ${ }^{4}$ Therefore, the ability of the patients to adhere to the instructions and related BPSM recommendations is very important. Only measurements following the recommendations are considered reliable. ${ }^{1-3}$ Thus, nonadherent patient behavior could lead to potential misdiagnoses and possibly result in inappropriate medication.,

Previously, we have suggested designing adherence models for sensing and modeling patient adherence during self-measurements using context-aware technology. ${ }^{7,8}$ The main purpose of these efforts was to model the adherence of the patient to the BPSM healthcare process. This was done using the adherence strategy engineering framework. ${ }^{9-11}$ As a result of this work, we have constructed a research prototype system, ValidAid, an automatic context-aware system for capturing BPSM data combined with the contextual data relating to selected recommendations. The ValidAid research system prototype is intended to help us investigate the domain and to provide a platform for developing more reliable systems for supporting unsupervised BPSM.

The aim of this study was twofold: to investigate patient adherence to self-reporting of BP data, as well as measuring the patient adherence to instructions given and the BPSM recommendations in the BPSM Room. 


\section{Patients and Methods PARTICIPANTS}

We included all patients self-monitoring in the BPSM Room during a period of 35 days. The participating patients suffered from chronic kidney disease or essential hypertension, and the majority (80-85\%) were receiving one or more antihypertensive medications.

We replaced the existing BPSM device in the BPSM Room with our ValidAid research prototype system, an automatic context-aware system for capturing BP data combined with relevant contextual data on patient adherence to selected recommendations. Patients were not informed of the experiment, in order not to interfere with their usual BPSM routine. ValidAid was configured to show the time of day as white text on a black background, and patients were told to write down all measurements from the BP device, along with the date and time of each measurement, as provided on the screen.

\section{THE BPSM ROOM}

The Department of Renal Medicine at Aarhus University Hospital receives more than 3,000 different patients each year in the outpatient clinic. About one-third of these are required to self-measure their BP. The decision whether a patient is capable of managing BPSM is made by the nurses and based on the patient's cognitive and physical ability to comprehend and perform the procedure.

During the first visit at the clinic the patient is instructed in the correct BPSM procedure, and usually the first measurement is supervised by a nurse. After this the staff expects the patients to act autonomously and self-directed performing all succeeding measurements in the BPSM Room unsupervised. Besides the instructions given, there are also written instructions mounted on a wall poster.

\section{ADHERENCE MODEL}

We created an adherence model of the BPSM healthcare process in the BPSM Room. Such a model allows us to measure and quantify current adherence levels, as well as provide a basis for improving patient adherence through guides and aids. Also, the model may be used as a clinical decision support tool for the healthcare staff. ${ }^{12}$

Patients are instructed to follow a subset of the recommendations from the Danish Hypertension Society ${ }^{13}$ providing training for their patients on how to perform reliable BPSM. Instructions include the following: to rest at least $5 \mathrm{~min}$ prior to the first measurement, not to move or talk during measurements, sit with the back supported against the backrest of the chair, keep feet flat on the ground and legs not crossed, and not to talk during the measurement. Also, the arm should be supported (e.g., resting on the table), and the patient should use the correct cuff size. In accordance with the Danish Hypertension Society guidelines, patients are instructed to take three consecutive measurements. Several related international guidelines exist on how to obtain reliable BP self-measurements, including those from the American Heart Association and the European Society of Hypertension. ${ }^{5,14}$ These recommendations exhibit minor differences, but share a common set of guidelines, comparable with the Danish Hypertension Society guidelines used in the BPSM Room.
Based on the above input, we designed an adherence model that models the relevant contextual information and supports using context-aware information technology for recording and assessing whether the patient is adhering to these guidelines.

\section{ADHERENCE STRATEGY}

An adherence strategy is defined as "the means, measures, and interventions used to facilitate patient adherence during a healthcare process as part of the overall disease management."12

For the BPSM Room, the main aim was to investigate whether patients would adhere to the recommendations of performing BPSM or not, as well as whether the patients were able to correctly selfreport BP measurements. Thus, an adherence strategy was defined, consisting of five adherence verifiers ${ }^{12}$ to update the adherence model with relevant context parameters.

The chosen adherence strategy combines the context data from the five verifiers and uses these to classify a measurement as being either adherent or nonadherent. Thus, the developed adherence strategy does not aim at improving adherence of the patient by exerting direct influence on the patient during the BPSM process, but rather on providing an assessment of the patient's adherence level as a telemedicine clinical decision support tool. This assessment may then be used to identify patients in need of additional instruction and training.

\section{VALIDAID RESEARCH PROTOTYPE SYSTEM OVERVIEW}

The ValidAid system consists of a clinically approved BP device as used in current clinical praxis, a sensor chair implementing four adherence verifiers for recording legs crossed, back supported, noise level, and rest time, respectively, and a table-based touch screen computer that integrates the BP device and sensor chair components, provides a user interface for the test facilitator, and performs audio classification of the data obtained (Fig. 1).

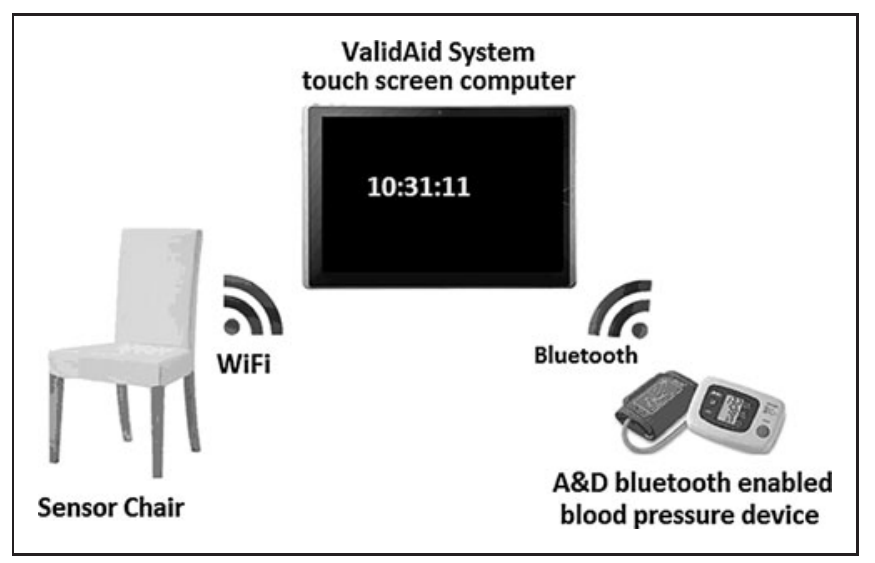

Fig. 1. When a test subject is seated, the ValidAid system monitors activity and posture and starts recording audio data. This occurs without the knowledge of the patient in order not to bias the experiment. When a blood pressure measurement is received, the audio data are processed and classified as speech or silence, and the adherence model is updated. 


\section{WAGNER ET AL.}

Also, all BP measurement data and contextual meta-data are collected in a structured format using extensible markup language (XML), ${ }^{15}$ and all audio data are stored as raw audio files for later review. ${ }^{16}$ Finally, all data are sent to a secure Web server using Web service technology in order to provide access to the data for the healthcare staff. ${ }^{17}$ The data can be accessed using a Web page as shown in Figure 2, which provides all primary data on the BP itself, as well as the raw contextual data and the calculated adherence level of the patient.

We use the sensor chair and associated software components in order to record test subjects getting seated and later standing up. This is used to determine when to start recording audio data and when a measurement session should be considered complete. Also, the sensor chair will provide information on patient rest time, whether the patient's back was supported, the ambient noise level in the room, and whether legs were crossed during measurement.

We have used the Reliable Evaluation Infrastructure (RELEI) framework for the main system architecture and design. ${ }^{18}$ RELEI provides us with additional tools for adherence engineering and patient adherence evaluation, as well as a reliable execution environment for the ValidAid application.

\section{COMPONENTS USED}

The touch screen computer ${ }^{19}$ is running the ValidAid application, which communicates with the BP device and sensor chair devices and also provides audio context classification capabilities. The audio classification algorithm is based on artificial neural network classification technology. ${ }^{20}$ Using an audio classification algorithm for detecting talk during measurement has previously been validated in a laboratory study ${ }^{21,22}$ and at an obstetrics department in a related study. $^{23}$

Our BP measuring device is the widely used A\&D UA-767PBT device, ${ }^{24}$ which can communicate wirelessly using Bluetooth ${ }^{\circledR}$. This device is specifically developed for telemedicine purposes and has previously been used for telemedicine studies involving BPSM. ${ }^{25}$

The sensor chair has previously been verified for sensing user posture during BPSM. ${ }^{11}$ The sensor part of the chair is based on an

\begin{tabular}{|cccccccc|}
\hline Date & Patient & $\begin{array}{c}\text { Adherence Systolic } \\
\text { Approved } \\
\text { mmHg }\end{array}$ & $\begin{array}{c}\text { Diastolic } \\
\mathrm{mmHg}\end{array}$ & $\begin{array}{c}\text { Pulse } \\
\text { BPM }\end{array}$ & $\begin{array}{c}\text { Time Seated } \\
\text { seconds }\end{array}$ & $\begin{array}{c}\text { Noise } \\
\mathrm{dB}\end{array}$ \\
\hline 09-11-2012 09:46:48 Unknown & $\square$ & 140 & 66 & 73 & 263 & 42,6 \\
09-11-2012 09:47:53 Unknown & & 130 & 61 & 72 & 328 & 51,6 \\
09-11-2012 09:48:48 Unknown & -126 & 61 & 70 & 383 & 33,2 \\
09-11-2012 09:52:22 Unknown & $\square$ & 122 & 81 & 99 & 103 & 50,2 \\
09-11-2012 09:53:02 Unknown & 114 & 80 & 98 & 144 & 44,8 \\
09-11-2012 09:53:40 Unknown & $\square$ & 110 & 80 & 96 & 181 & 48,6 \\
09-11-2012 09:54:18 Unknown & 118 & 86 & 99 & 219 & 48,6 \\
& & & & & & & \\
\hline
\end{tabular}

Fig. 2. An excerpt from the Web solution that provides healthcare staff access to the self-measured data from the Blood Pressure Self-Measurement Room. For each patient, all blood pressure data and contextual data are provided, as well as an indication of whether the measurement was valid (approved) or not. BPM, beats per minute. embedded Linux Single Board Computer prototyping platform ${ }^{26}$ using two external piezoresistive sensors ${ }^{27}$ for sensing leg placement and user seated. Also, a pressure sensor ${ }^{28}$ placed in the backseat of the chair provides context information of whether the patient is seated with the back supported. Finally, a noise sensor ${ }^{29}$ was built into the chair for providing a continuous decibel measurement of the ambient background noise.

\section{DATA COLLECTION}

We deployed the ValidAid system in the BPSM Room for the duration of 35 days. We automatically and anonymously included all patients who used our BP device and sat in the sensor chair. Participants were not informed about the ValidAid system and the experiment, and if anyone asked, the patients were instructed not to deviate from their usual practice and proceed as usual. The usual procedure include filling out a paper schema with date and time, as well as three measurements each consisting of a systolic and diastolic $\mathrm{BP}$ parameter and the heart rate. For each participant, the ValidAid system recorded their ability to follow the guidelines before and during measurement. Specifically, the system autonomously collected data on the context and patient behavior, as well as the BP measurements obtained from the BP device.

\section{DATA ANALYSIS}

During the 35-day study period we collected the paper schemas filled out by the participants. We then matched all the self-reported data with the data recorded by the ValidAid system, based on a combination of date and time. Following this, we compared the selfreported data with the data recorded by the ValidAid system, in order to identify any error in patient reporting. Then, we analyzed the recorded context behavior data with regard to the patients' ability to adhere to the instructions provided to them. Finally, we applied descriptive statistics to the data, in order to discover relevant trends and themes.

\section{Results}

\section{SELF-REPORTING ADHERENCE}

We collected a total of 646 measurements. We managed to identify 113 patients from the collected paper schemas, constituting 363 of the 646 measurements. The remaining measurements belonged to patients for whom the paper schemas were either mislaid in the clinic by staff or patients or not possible to identify because of bad handwriting, wrong date or time, or other reporting errors.

Of the 113 identified patients, 59 (52.2\%) had performed exactly three BP measurements, reported these data accurately, and provided the correct time and date. A further 15 patients (13.3\%) had also performed exactly three BP measurements and reported these data accurately but did not provide the correct time or date.

In total, 74 patients $(59+15$ [65.5\%]) had self-measured the required three BP measurements and reported the correct 
measurements compared with the automatically recorded data in the ValidAid system.

The remaining 39 patients (34.5\%) failed to correctly selfreport the measured data. Of these, 23 (20.4\%) had in fact performed more than the required three measurements, ranging from one to five additional measurements, without reporting these on paper, and selecting three of the measurements to selfreport, typically those with the lowest BP and heart rate values.

Furthermore, 16 patients (14.2\%) had omitted, changed, and/ or fabricated measurements. This included anything from changing one or all data parameters of a measurement. Table 1 gives an overview.

\section{PATIENT ADHERENCE TO RECOMMENDATIONS}

Of the 113 identified patients, only $8 \%$ adhered to the required rest time before taking the first measurement. On average, the 113 patients waited 2 min $11 \mathrm{~s}$ before taking the first measurement. In Figure 3 an overview is provided of patient rest time grouped in 30-s intervals.

Concerning the full 646 measurements recorded during the 35-day period, we excluded four measurements from the study because of participant error. In all four excluded measurements, the rest time was recorded as zero, which is only possible if the participant was not seated in the sensor chair during measurement, for example, standing up during measurement or sitting on the table or elsewhere.

In analyzing the 642 valid measurements, including the first, second, third, and following measurements of the individual participant, the mean rest time before commencing the measurements was $4 \mathrm{~min}$ $13 \mathrm{~s}$ (median, $3 \mathrm{~min} 27 \mathrm{~s}$ ). This implies that less than half of all measurements were performed within the required 5-min rest time.

When analyzing the overall ability of participants to adhere to the recommendations investigated in this study, also including "Not talking," "Legs not crossed," "Back supported," and "Quiet settings," we found that none of the participants $(0 \%)$ followed all of the five recommendations we investigated. Most participants avoided talking during measurement (99.1\%), and a majority avoided high ambient

Table 1. Patients' Ability to Self-Report Divided

into Thematic Groups of Both Adherent and Nonadherent Patients Including Type of Error

SELF-REPORTING NUMBER

ADHERENCE OF PATIENTS OF PATIENTS PERCENTAGE

\begin{tabular}{l|c|c}
\hline All data reported correctly & 59 & $52.2 \%$ \\
\hline $\begin{array}{l}\text { Blood pressure reported correctly, } \\
\text { date/time missing }\end{array}$ & 15 & $13.3 \%$ \\
\hline $\begin{array}{l}\text { Extra measurements beyond } \\
\text { the required three }\end{array}$ & 23 & $20.4 \%$ \\
\hline $\begin{array}{l}\text { Data reported incorrectly or missing, } \\
\text { or too few measurements }\end{array}$ & 16 & $14.2 \%$ \\
\hline Identified patients, total & 113 & $100.0 \%$ \\
\hline
\end{tabular}

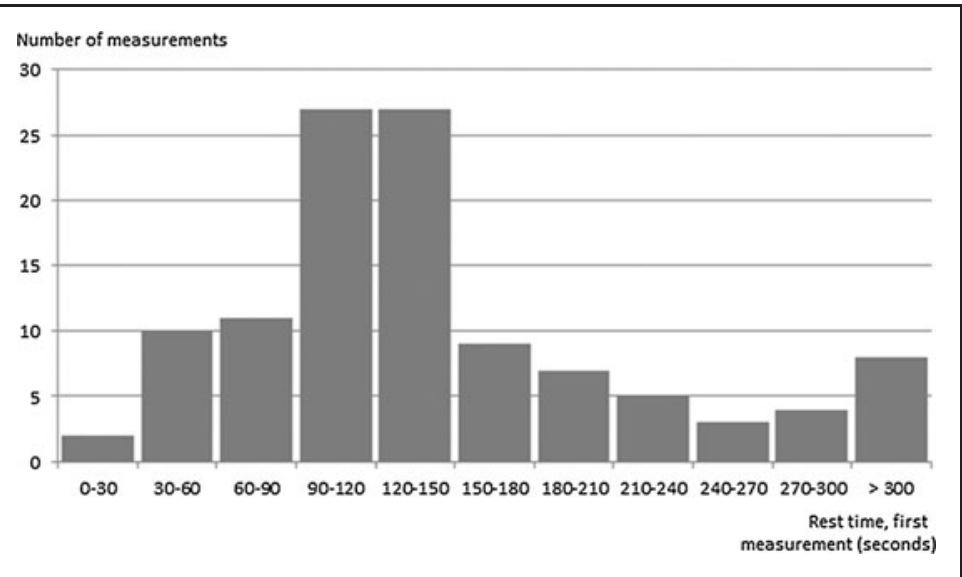

Fig. 3. Rest time before first measurement grouped in 30-s intervals All measurements with a rest time of $300 \mathrm{~s}$ were grouped together, as these constitute adherence to the 5 -min rest time.

background noise (90.0\%). However, only a third of the measurements were in compliance with the 5-min rest time before the measurement, and $43.5 \%$ complied with legs not crossed, whereas only $5 \%$ sat correctly with their back supported. The detailed results can be found in Table 2.

Finally, in Table 3 we present an overview of the total number of individual measurements that adhered to between none to five of the recommendations investigated in this study.

\section{Discussion \\ CHALLENGES RELATED TO SELF-REPORTING}

We found that over a third (34.5\%) of the participants failed to self-report accurately, either omitting, doubling, rounding, or even fabricating one or more parameters in one or more of their measurements. We can only speculate on the motives for doing so: embarrassment, self-denial, or because of simple operator errors, bad eyesight, or low concentration. Irrespective of the reason, it represents a challenge to the validity of the data being self-reported.

Table 2. Overview of the Number of Measurements

That Adhered to Each of the Five Recommendations,

Shown as Number of Measurements and Percentages

\section{MEASUREMENTS ADHERING TO}

\begin{tabular}{l|c|c}
\multicolumn{1}{c|}{ Not talking } & 636 & $99.1 \%$ \\
\hline Quiet settings & 578 & $90.0 \%$ \\
\hline Legs not crossed & 278 & $43.3 \%$ \\
\hline Rest time & 187 & $29.1 \%$ \\
\hline Back supported & 32 & $5.0 \%$ \\
\hline All five recommendations & 0 & $0.0 \%$ \\
\hline
\end{tabular}


Table 3. Number of Recommendations Adhered to by Number of Individual Measurements and the Percentage of Total Measurements NUMBER OF NUMBER OF RECOMMENDATIONS MEASUREMENTS

\begin{tabular}{l|c|c}
\hline 0 & 0 & $0.0 \%$ \\
\hline 1 & 26 & $4.0 \%$ \\
\hline 2 & 242 & $37.7 \%$ \\
\hline 3 & 296 & $46.1 \%$ \\
\hline 4 & 77 & $12.0 \%$ \\
\hline 5 & 0 & $0.0 \%$ \\
\hline
\end{tabular}

Securing the highest data quality is crucial to the treating physicians' ability to perform the correct diagnosis and interventions. ${ }^{2,14,30}$

The findings of reduced self-reporting compliance are in line with previous work in the area. ${ }^{31-33}$ In these studies patients were equipped with home BP devices but were not informed that the devices were capable of storing the measurements, in order to examine participants' ability to correctly self-report measurements. After a period of self-monitoring filling out paper records, these records were compared with BP device memory values. In total, more than half the patients had either omitted or fabricated readings, indicating unacceptable levels of reporting bias. In a later study, Santamore et al. ${ }^{34}$ investigated the ability of 161 patients to accurately report selfmeasured BP data using a telemedicine Web solution interface and a home BP device. The study compared the self-reported data from the Web with the data stored in the device memory. The authors found that around $16 \%$ of the reported data deviated from the actual data stored in the device memory. Also, Santamore et al. ${ }^{34}$ found that the average error was below $4 \mathrm{~mm} \mathrm{Hg}$ and thus not of major importance to the prognostic value for diagnostic or monitoring purposes. The lower error rate reported in this previous study compared with both our findings and the findings in the other three reported studies ${ }^{31-33}$ could be due to participants entering data into a Web solution rather than keeping a paper log. This implies that the participants were aware of technology being involved and thus presumably less likely to be tempted to misreport. Also, as we cannot expect all patient types to be able to use a Web solution for self-reporting of data, it could indicate that the study of Santamore et al. ${ }^{34}$ included a population with higher competencies than was the case in our study as well as in the other three related studies. ${ }^{31-33}$

None of the four studies ${ }^{31-34}$ investigated adherence to the recommendations, such as rest time before measurement, talking, and noise levels, focusing solely on the ability to correctly and accurately perform and self-report BPSM data. However, the telemedicine solution discussed by Santamore et al. ${ }^{34}$ did provide the means to selfreport the amount of cigarettes smoked.

Regarding further differences, our study took place at a highly specialized department where participants are carefully instructed and motivated by staff to report correctly, rather than in the home setting as was the case in the four related studies.

Combining the results from the related studies with our own findings provides us with a clear indication of the challenges related to relying on BPSM obtained in the unsupervised setting with regard to patients' ability to accurately report self-measured data. In conclusion, self-reported data cannot be trusted to be accurate with currently available technology.

\section{PATIENT ADHERENCE TO THE RECOMMENDATIONS}

We found that only $8 \%$ of the patients adhered to the required rest time before taking the first measurement; less than half of all measurements, including the second and third measurement, were performed after the required 5-min rest time. Rest time is considered one of the critical parameters and could cause unacceptable bias to the measurement if not properly adhered to. ${ }^{2}$

When analyzing the overall ability of participants to adhere to the recommendations investigated in this study, including "Not talking," "Legs not crossed," "Back supported," and "Quiet settings," we found that none of the participants followed all of the five recommendations. Most participants avoided talking and avoided excessive ambient background noise. However, only a third of the measurements were in compliance with the 5-min rest time before the measurement and in compliance with legs not crossed, whereas most participants did not have their back correctly supported during measurements.

Not complying with just a single of the above recommendations may create significant bias to the measurement, in effect rendering the data unusable or even harmful. ${ }^{1,2,35}$ As no single participant was able to follow all of the five measured recommendations, only a minority adhered to four out of five, and less than half adhered to two out of five, this indicates a serious challenge to current BPSM praxis in the BPSM Room.

Focusing on patient context in order to verify the quality of the measurement has not been investigated sufficiently in the existing literature. Only a few studies have focused on context, and within BPSM only Copetti et al. ${ }^{36}$ and D'Angelo et al. ${ }^{37}$ have suggested systems for ambulatory BPSM (24-h BP measurement) enriched with context data. However, neither of these studies has been evaluated in the clinic with patients. Copetti et al. ${ }^{36}$ relied on smarthome sensor technologies for sensing user activities during ambulatory BP measurement to build a context-aware telemonitoring system; however, they targeted the home environment and not the clinic. D'Angelo et al. ${ }^{37}$ presented a system for motion-aware ambulatory BP measurement. They focused on measurement errors occurring due to movement artifacts, wrong posture during the measurement, or absence of proper rest time prior to the measurement. They suggested sensing motions and postponing planned measurements if proper rest is not detected. Additionally, their research prototype detects whether the user is in the proper upright position during measurement. As such, like in our study, D'Angelo et al. ${ }^{37}$ were also concerned with patient adherence to the recommendations, as well as building adherence verifiers and aids to support more reliable data. 


\section{MEASUREMENT ADHERENCE}

Our own group has previously deployed the ValidAid system in the clinical setting in the waiting room of an obstetrics department. ${ }^{23}$ Here, we observed 81 pregnant diabetic women self-measuring BP while preparing for their weekly or biweekly medical consultation. Of these, 41 used the ValidAid system for BPSM. The study found that the pregnant diabetic women predominantly did not adhere to given instructions when performing BPSM in the waiting room and that the ValidAid research prototype system was able to 100\% accurately model this nonadherent behavior. ${ }^{23}$

Within the field of telemedicine, several state-of-the-art platforms exist that also feature BP measurement and automatic data collection in order to avoid reporting errors. This includes the Intel Health Guide (IHG), which has been used in several telemedicine studies. ${ }^{38,39}$ The IHG allows the patient to take the recommended three successive measurements and then automatically calculates the average, thus enforcing correct reporting procedure eliminating the risk of reporting bias. The IHG also features the capability of enforcing a 1min wait between the three measurements as recommended in most guidelines. However, the system does not have any context-aware sensors and cannot check whether the patient has remained silent during measurements or observed the proper rest time. It does feature a range of interactive questionnaires, allowing the user to self-report whether he or she has rested sufficiently, been drinking coffee, or smoking cigarettes. Similar systems include the Tunstall Mymedic ${ }^{40}$ and the Bosch Health Buddy, ${ }^{41}$ both of which feature automatic collection of BP data and interactive questionnaires, thus avoiding reporting errors but still relying on self-reporting for relevant context. However, none of these three systems supports any of the recommendations investigated in this study.

\section{OBSERVER BIAS}

Our study was planned to be unobtrusive in order to prevent any form of observer bias. This was achieved through an automatic installation that did not require an experiment facilitator to be present. However, the presence of the sensor chair and the touch screen computer and the change of BP device from the usual model may have alerted some patients. Thus, it is possible that some participants had changed their behavior during their respective BPSM healthcare processes. In order to remedy this potential for bias, we need an experimental setup that is completely indistinguishable from the normal equipment in the BPSM Room.

\section{SUGGESTIONS FOR THE CLINIC}

Our results provide a strong indication that the current praxis is not an adequate method for providing reliable BP measurements for all patients in the BPSM Room. As a consequence, we suggest that further training or guidance should be provided to achieve a higher degree of patient self-measurement adherence. As the current level of patient instruction is already very high, further instructions and training may not be adequate. Rather, we suggest adding technology support that would allow us to target the nonadherent individuals.

Furthermore, the ValidAid system deployed in the BPSM Room is capable of identifying individual patients using a small radio iden- tification card (RFID), ${ }^{42}$ barcode identification (QR code), ${ }^{43}$ and alternatively using face recognition. ${ }^{44}$ All of these are provided as part of the RELEI framework. ${ }^{18}$ Such technology, or other means of participant identification, would allow the treating physician or nurse to be alerted to specific patients who have not adhered to the recommendations in part or full, thus marking them for additional training and instructions, using a suitable interface for the healthcare professionals (e.g. as part of the electronic health record system). Such a system might be of most value if it could automatically identify nonadherent patients and inform the healthcare professionals during the ensuing consultation.

Alternatively, we suggest introducing increased tool support in the clinic, for providing improved guidance to patients while selfmeasuring (e.g., correcting any user nonadherent behavior in real time rather than retrospectively). These objectives could be achieved by basing such a system on the adherence verifiers created for the ValidAid system and extending these with proper adherence aids for guiding the user. Adherence aids can be considered "the gold standard" for improving patient adherence, and it could be argued that adherence aids should be prioritized in adherence strategy engineering projects. ${ }^{12}$ However, adherence aids have proven to be more complex to design and implement and are much more intrusive in the healthcare process. Relying, for example, on an audio- or videobased guiding system might be disturbing in the BPSM Room. Thus, this option should be implemented with caution, as adherence aids could be the cause of unacceptable bias during BPSM. This could potentially lead to adverse effects not unlike the "white coat effect," as the increased computer presence may affect the patients' psychological state and increase the BP. ${ }^{4}$

Finally, a major downside to context-aware systems is the complexity of the individual systems and the cost of deployment and maintenance. Any benefits resulting from such a system should be carefully weighed against the increased cost and time consumed.

\section{Future Work}

Although our results provide a good indication of the challenges facing hypertensive patients performing BPSM in the BPSM Room, it would be beneficial to obtain more data on actual use in the current clinical praxis. This could be achieved by creating a permanent and ubiquitous installation of the ValidAid research prototype system, preferably covering all attending patients. This would provide us access to a larger patient population, which would allow us to draw more detailed conclusions. Contributing to this, the studies could be repeated at more sites, including hospitals and other clinical settings, in order to understand how general the problem is. Also, the problem is likely to be of an equal or larger scale in the unsupervised home setting of self-measuring patients.

Furthermore, the current reporting format for the healthcare staff of the individual patient adherence level as presented in Figure 2 could be refined to better support staff to identify nonadherent patients in need of more training and instructions. This would require secure identification of patients, which is already supported in the RELEI framework. 
Also, it would be relevant to create a research prototype that would act as an adherence aid, guiding the user through the measurement process in order to investigate whether this could improve adherence without causing any adverse effects. This study could be planned as a blinded randomized study with three patient groups: one with the BP device used in current praxis, one group using a pure adherence verifier solution, and one group using an adherence aid solution for guidance during the measurement process in order to also discover long-term trends in BP treatment outcomes.

Patients underreporting their true BP are in risk of becoming undermedicated. This could lead to a further deterioration in their health condition and higher mortality. On the other hand, patients failing to adhere to recommendations (e.g., because of insufficient rest time) are at risk of getting overmedicated as their BP is measured as being erroneously high. This leads to added medical costs to the patients, as well as exposing them to the adverse effects of hypertensive medications. ${ }^{45}$

However, although patient adherence to the recommendations appears relevant to investigate further, hard end points for the measuring the effect in terms of disease progression and mortality rates requires follow-up of a large population for several years.

Finally, we find it relevant to investigate additional BPSM recommendations, and patient adherence to them, including the feasibility of detecting adherence to arm support and correct cuff size. Other contextual factors are known to influence BP, including ambient room temperature. Also, patient activities such as having recently smoked, eaten, and ingested coffee prior to the measurement are known to have an effect on BP and thus relevant to investigate. ${ }^{1,2,5}$ Although some of these parameters might be easy to record using state-of-the-art sensor technologies (e.g., ambient room temperature), others might require the user to self-report them. However, as indicated by the results of this study, self-reporting cannot be trusted in full.

\section{Conclusions}

We obtained 646 individual BP measurements and related context datasets, of which 642 were used to investigate patient adherence to the recommendations. We also obtained from 113 unique patients the self-reported data that we compared with the data automatically obtained by the ValidAid system. We found clear indications that the current praxis for BP self-measurement utilized in the BPSM Room is facing several challenges regarding the reliability of self-measured and self-reported BP data. Existing BP devices are not capable of addressing these challenges, and patients are to a large extent not capable of accurately reporting the data. Instead, we should rely on implementing a new class of context-aware clinical support systems for use in the BPSM Room and similar clinical self-measurement locations, which are able to pinpoint nonadherent behavior and alert the staff or guide the patients to be more compliant to the recommendations. This could be achieved through the use of targeted adherence engineering following the adherence strategy engineering framework methodology.

We also found that the adherence model constructed as part of the ValidAid research prototype system could effectively model the nonadherent behavior of the patients included in the study. As such, we consider the ValidAid system to be a relevant and successful example of applying context-aware technology to the clinical unsupervised setting.

The ValidAid system is generally applicable to other selfmeasurement environments, including the home setting and remote outpatient clinics, as it is built using telemedicine technology and thus well suited for remote monitoring and diagnosis.

Finally, in order to determine the significance and clinical impact of introducing a novel BPSM method, as proposed with the ValidAid system, further investigations for measuring the hard end points effect in terms of disease progression and mortality rates are required.

\section{Acknowledgments}

Thanks to Dr. Kent Lodberg Christensen from the Department of Cardiology, Aarhus University Hospital, for assistance with the initial understanding on hypertension and blood pressure measurement techniques and best practices. Thanks to the head nurse Lone Stougaard and other medical staff at the Department of Renal Medicine for providing valuable support for the project. Thanks to industrial designer Anders Bech Christensen, for assisting with the conceptual design of the sensor chair and software graphics. Thank you to Torben Lund Jensen and Rasmus Elm Sørensen at the University Electronics and Mechanics Workshop for work on the prototype hardware.

\section{Disclosure Statement}

No competing financial interests exist.

\section{REFERENCES}

1. Campbell NRC, McKay DW. Accurate blood pressure measurement: Why does it matter? CMAJ 1999;161:277-278.

2. Pickering TG. Ambulatory monitoring and blood pressure variability. London: Science Press, 1991.

3. Pierdomenico SD, Di Nicola M, Esposito AL, Di Mascio R, Ballone E, Lapenna D, et al. Prognostic value of different indices of blood pressure variability in hypertensive patients. Am J Hypertens 2009;22:842-847.

4. Wagner S, Toftegaard TS, Bertelsen OW. Challenges in blood pressure selfmeasurement. Int J Telemed App/ 2012;2012:437350.

5. Pickering TG, Miller NH, Ogedegbe G, Krakoff LR, Artinian NT, Goff D, et al. Call to action on use and reimbursement for home blood pressure monitoring: Executive summary: A joint scientific statement from the American Heart Association, American Society of Hypertension, and Preventive Cardiovascular Nurses Association. Hypertension 2008;52:1-9.

6. AbuDagga A, Resnick HE, Alwan M. Impact of blood pressure telemonitoring on hypertension outcomes: A literature review. Telemed J E Health 2010;16:830838

7. Weiser M. Some computer science issues in ubiquitous computing. Commun ACM 1993;36:75-84.

8. Dey AK. Context-aware computing. In: Krumm J, ed. Ubiquitous computing fundamentals. Boca Raton, FL: CRC Press, 2010:321-353.

9. Wagner S, Toftegaard TS, Bertelsen OW. Novel approach for ensuring increased validity in home blood pressure monitoring. 4th Int Conf Pervasive Comput Technol Healthc (PervasiveHealth) 2010:1-4.

10. Wagner S, Toftegaard TS, Bertelsen OW. Increased data quality in home blood pressure monitoring through context awareness. 5th Int Conf Pervasive Comput Technol Healthc (PervasiveHealth) 2011:234-237. 


\section{MEASUREMENT ADHERENCE}

11. Wagner S, Toftegaard T, Bertelsen 0 . Context assessment during blood pressure self-measurement utilizing the sensor chair. Ambient Intell 2011:295-299.

12. Wagner S, Toftegaard TS, Bertelsen OW. Introducing the adherence strategy engineering framework (ASEF). Support for developing technology-based selfcare solutions. J Methods Inf Med 2013 (in press). doi: 10.3414/ME12-01-0027.

13. Bang LE, Christensen KL, Hansen KW, Skov K, Wiinberg N. Diagnostisk blodtryksmåling-på døgnbasis, hjemme og i konsultationen. Available at www.dahs.dk/fileadmin/ BTmaaling_version-17.pdf (last accessed February 16, 2013).

14. O'Brien E, Asmar R, Beilin L, Imai Y, Mallion JM, Mancia G, et al. European Society of Hypertension recommendations for conventional, ambulatory and home blood pressure measurement. J Hypertens 2003;21:821-848.

15. Bray T, Paoli J, Sperberg-McQueen CM, Maler E, Yergeau F. Extensible markup language (XML) 1.0. Available at www.w3pdf.com/W3cSpec/XML/2/REC-xml1120060816.pdf (last accessed April 9, 2013).

16. Microsoft Inc. Multiple channel audio data and WAVE files. 2013. Available at http://msdn.microsoft.com/en-us/windows/hardware/gg463006.aspx (last accessed January 1, 2013).

17. Curbera F, Duftler M, Khalaf R, Nagy W, Mukhi N, Weerawarana S. Unraveling the Web services web: An introduction to SOAP, WSDL, and UDDI. Internet Comput IEEE 2002;6:86-93.

18. Wagner S, Toftegaard TS, Bertelsen OW. Requirements for an evaluation infrastructure for reliable pervasive healthcare research. 6th Int Conf Pervasive Comput Technol Healthc (PervasiveHealth) 2012:260-267.

19. ASUSTek Computer Inc. Eee Slate EP121. Available at www.asus.com/Eee/ Eee_Pad/Eee_Slate_EP121/(last accessed January 1, 2012).

20. Bishop CM. Pattern recognition and machine learning. New York: Springer, 2006.

21. Wagner S, Rasmussen NH, Ahrendt P, Toftegaard TS, Bertelsen OW. Context classification during blood pressure self-measurement using the sensor seat and the audio classification device. 6th Int Conf Pervasive Comput Technol Healthc (PervasiveHealth) 2012:201-202.

22. Wagner S, Ahrendt P, Toftegaard TS, Bertelsen OW. Audio context classification for determining blood pressure self-measurement adherence. Proc IADIS Int Conf e-Health 2012:105-114.

23. Wagner S, Kamper, C H, Rasmussen, NH, Ahrendt P, Toftegaard TS, Bertelsen OW. Reliable blood pressure self-measurement in the obstetric waiting room. $J$ Methods Inf Med 2013 (submitted for publication).

24. A\&D Medical Pty Ltd. UA-767 semi automatic blood pressure monitor. Available at www.andmedical.com.au/web.php?p=1201\&pp =\&pcat = aibpm (last accessed January 17, 2012).

25. Huniche L, Dinesen B, Grann O, Toft E, Nielsen C. Empowering patients with COPD using tele-homecare technology. Stud Health Technol Inform 2010;155:48-54.

26. Phidgets Inc. Phdiget SBC2 product page. Available at www.phidgets.com/ products.php?category $=21 \&$ product_id $=1072 \_0,1 / 1 / 2012$ (last accessed January 1, 2012).

27. Tekscn Inc. FlexiForce. Load/force sensors and ELF: Economical load and force measurement systems. Available at www.tekscan.com/flexiforce.html (last accessed January 1, 2012).

28. Premier Farnell UK Ltd. Defender-PM1/PK-Pressure mat. Available at http:// uk.farnell.com/defender-security/pm1-pk/pressure-mat-stair-595×170mm/dp/ 1146761 (last accessed January 1, 2012).

29. Phidgets Inc. Sound sensor. Available at www.phidgets.com/ products.php?category $=6 \&$ product_id $=1133 \_0$ (last accessed January 1, 2012).

30. Pickering TG, White WB, Giles TD, Black HR, Izzo JL, Materson BJ, et al. When and how to use self (home) and ambulatory blood pressure monitoring. J Am Soc Hypertens 2010;4:56-61.

31. Johnson KA, Partsch DJ, Rippole LL, McVey DM. Reliability of self-reported blood pressure measurements. Arch Intern Med 1999;159:2689-2693.
32. Mengden T, Hernandez Medina RM, Beltran B, Alvarez E, Kraft K, Vetter $H$. Reliability of reporting self-measured blood pressure values by hypertensive patients. Am J Hypertens 1998;11:1413-1417.

33. Myers MG. Self-measurement of blood pressure at home: The potential for reporting bias. Blood Press Monit 1998;3(Suppl 1):S19-S22.

34. Santamore WP, Homko CJ, Kashem A, McConnell TR, Menapace FJ, Bove AA. Accuracy of blood pressure measurements transmitted through a telemedicine system in underserved populations. Telemed J E Health 2008;14:333-338.

35. Campbell NR, Chockalingam A, Fodor JG, McKay DW. Accurate, reproducible measurement of blood pressure. CMAJ 1990;143:19-24.

36. Copetti A, Loques O, Leite JCB, Barbosa TPC, da Nobrega ACL. Intelligent context-aware monitoring of hypertensive patients. 3rd Int Conf Pervasive Comput Technol Healthc (PervasiveHealth) 2009:1-6.

37. D'Angelo LT, Lohmann M, Lueth TC. A new device for motion-aware ambulatory blood pressure measurement. 5th Int Conf Pervasive Comput Technol Healthc (PervasiveHealth) 2011:17-23.

38. Intel Corporation. Intel Health Guide PHS6000. Available at www.intel.com/ corporate/healthcare/emea/eng/healthguide/pdfs/Health_Guide_Product_ Brief.pdf (last accessed January 1, 2011).

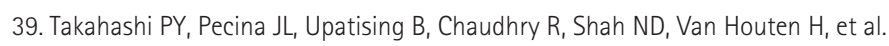
A randomized controlled trial of telemonitoring in older adults with multiple health issues to prevent hospitalizations and emergency department visits. Arch Intern Med 2012;172:773-779.

40. Tunstall Ltd. Telehealth solutions. Available at www.tunstall.co.uk/Our-products/ Telehealth-solutions (last accessed January 1, 2011).

41. Koff $P$, Jones RH, Cashman JM, Voelkel NF, Vandivier R. Proactive integrated care improves quality of life in patients with COPD. Eur Respir J 2009;33: 1031-1038.

42. ThingMagic RFID readers help enhance patient experience at California cancer center. Available at www.thingmagic.com/press-room/27-press-releases/285thingmagic-rfid-readers-help-enhance-patient-experience-at-californiacancer-center (last accessed November 16, 2011).

43. Baik S. Rethinking QR code: Analog portal to digital world. Multimedia Tools App/ 2012:1-8.

44. Wagner A, Wright J, Ganesh A, Zhou Z, Mobahi H, Ma Y. Toward a practical face recognition system: Robust alignment and illumination by sparse representation. IEEE Trans Pattern Analysis Machine Intell 2012;34:372-386.

45. Pickering TG, Hall JE, Appel $U_{1}$ Falkner BE, Graves J, Hill MN, et al. Recommendations for blood pressure measurement in humans and experimental animals: Part 1: Blood pressure measurement in humans: A statement for professionals from the Subcommittee of Professional and Public Education of the American Heart Association Council on High Blood Pressure Research. Circulation 2005;111:697-716. 